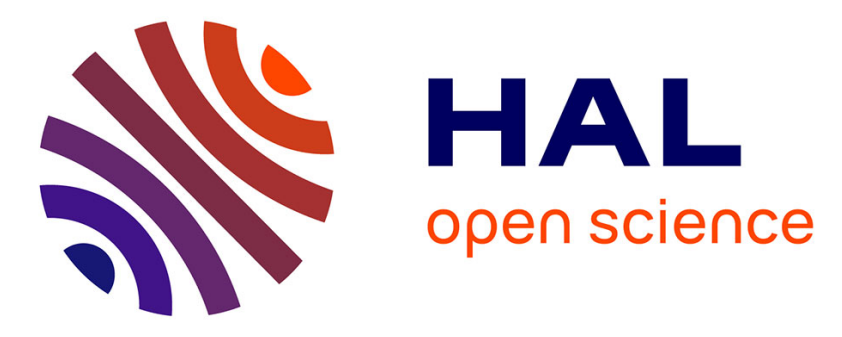

\title{
Localization of small objects with electric sense based on kalman filter
}

\author{
Vincent Lebastard, C Chevallereau, A Girin, Frédéric Boyer, Pol-Bernard
}

Gossiaux

\section{- To cite this version:}

Vincent Lebastard, C Chevallereau, A Girin, Frédéric Boyer, Pol-Bernard Gossiaux. Localization of small objects with electric sense based on kalman filter. 2012 IEEE International Conference on Robotics and Automation, 2012, Saint Paul, Minnesota, USA, United States. pp.1137 - 1142, 10.1109/ICRA.2012.6224824 . hal-01503057

\section{HAL Id: hal-01503057 \\ https://hal.science/hal-01503057}

Submitted on 6 Apr 2017

HAL is a multi-disciplinary open access archive for the deposit and dissemination of scientific research documents, whether they are published or not. The documents may come from teaching and research institutions in France or abroad, or from public or private research centers.
L'archive ouverte pluridisciplinaire HAL, est destinée au dépôt et à la diffusion de documents scientifiques de niveau recherche, publiés ou non, émanant des établissements d'enseignement et de recherche français ou étrangers, des laboratoires publics ou privés. 


\title{
Localization of small objects with electric sense based on kalman filter
}

\author{
V. Lebastard*, C. Chevallereau*, A. Girin*, F. Boyer* and P.B. Gossiaux ${ }^{\dagger}$
}

\begin{abstract}
Electric fish feel the perturbations of a selfgenerated electric field through their electro-receptive skin. This sense allows them to navigate and reconstruct their environment in conditions where vision and sonar cannot work. In this article, we use a sensor bio-inspired from this active sense to address the problem of small objects reconstruction and electrolocation. Based on a Kalman filter, any small object in the surrounding of the motion controlled sensor can be encapsulated into an equivalent sphere whose location is well estimated by the filter. Experimental results illustrate the feasibility of the approach.
\end{abstract}

\section{INTRODUCTION}

Developed by several hundreds of fish who have coevolved on both African and South-American continents, electric sense have been discovered by Lissman in 1958 [1]. In the African fish Gnathonemus Petersii for instance, the fish first polarizes its body with respect to an electric organ discharge (EOD) located at the basis of its tail (Fig. 1). This polarization which is applied during a short timepulse, generates around the fish a dipolar shaped electric field which is distorted by the objects present in its surroundings. Then, thanks to many electro-receptors distributed along its body, the fish "measures" the distortion of the electric fields and infers through a brain processing intensively studied by neurobiologists [2] an image of its surroundings. In order to study the environment modalities sensed by the fish, biologists have developed many neuro-ethological experiments in which fish are trained to recognize a given shape [3], [4]. More commonly, electric fish can easily navigate in the dark or/and turbid waters of confined unstructured environments as the roots of the trees of flooded tropical forests which are their natural habitat. Also, electric sense has a quite narrow but relevant niche since none other sense as vision or sonar can work in these conditions. Based on these potential interests, Mc. Iver and co authors have recently exploited an experimental set up of four point electrodes placed at the apexes of a rhombus in a rigid moving frame driven by a Cartesian robot [5]. In this device, two electrodes situated at the opposite apexes of the lozenge are polarized in voltage and play the role of the electric organ of discharge of the fish while the two opposite electrodes play the role of receivers

V. Lebastard is the corresponding author: E-Mail: vincent.lebastard@emn.fr, Tel.: +00 33251858308.

${ }^{*}$ F. Boyer, A. Girin and V. Lebastard are with IRCCyN-Ecole des Mines de Nantes, 4, rue Alfred Kastler B.P. 20722 - 44307 Nantes Cedex 3 France.

${ }^{\star} \mathrm{C}$. Chevallereau is with IRCCyN-CNRS, Ecole Centrale de Nantes, 1 rue de la Noë, 44000 Nantes, France.

$\dagger$ P.B. Gossiaux is with SUBATECH-Ecole des Mines de Nantes, 4, rue Alfred Kastler B.P. 20722 - 44307 Nantes Cedex 3. between which the tension is measured. Using the Cartesian motion control of this sensor in a tank, they successfully located small spheres through off-line particle filtering [6], [5]. However, the body geometry of this device ideally reduces to four points electrodes so limiting the implementation of its principle on a real autonomous underwater vehicle. Thus, using an alternative sensor technology based on the measurements of the currents, in [7], [8], Kalman filtering technics have been applied to the reconstruction of the surrounding of an underwater vehicle of realistic geometry. In [7] the surroundings is constituted of insulating walls and corners as those of a tank, while in [8], the case of small (insulating and conducting) objects is tackled. However, all these results have been till today restricted to simulations. Also, the article here presented aims at experimenting these solutions on a electric test-bed. Going further, the article here presented mainly addresses, in experimental conditions, the problem of the reconstruction of small objects. By "reconstruction", we mean the estimate of their size and their location in the sensor frame. Because the equations of electricity cannot be integrated analytically for an object of arbitrary shape [9], we reduce our investigations to the case of spherical objects. However, we will see that other shaped objects as the cube, can be modeled by (and encapsulated in) equivalent spheres.

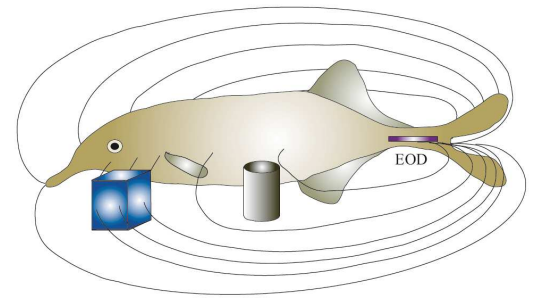

Fig. 1. The electric fish, here the Gnathonemus Petersii. The electric field is distorted by the presence of an object (for instance, an insulating cylinder blocks the field lines whereas a conductive cube funnels them).

The article is structured as follows. First we will briefly present the sensor technology (section II). Then, we address in section III the modeling of the sensor measurements generated by its surrounding. A solution to localize and reconstruct the small objects is detailed in section IV. It will be tested on an experimental test-bed in section $\mathrm{V}$. Finally, the article ends with some concluding remarks and perspectives in section VI. 


\section{THE SENSOR}

Here, we just remind the physical principle of the sensor and refer the reader to [10] for more details about its technology.

\section{A. The sensor principle}

From an external point of view, the sensor is constituted of a body with insulating wetted boundaries on which are fixed a set of $m+1$ electrodes noted $\mathcal{E}_{0}, \mathcal{E}_{1} \ldots \mathcal{E}_{m}$ (figure 2 ). Thanks to a tension generator, the potential on $\mathcal{E}_{\alpha=1,2 \ldots m}$ is imposed to be the common ground with respect to which $\mathcal{E}_{0}$ is put under the controlled tension $U$. Once the sensor immersed in a fluid, this active device produces a field of current lines flowing through the sensor surroundings from $\mathcal{E}_{0}$ to the other macro-electrodes $\mathcal{E}_{\alpha=1, \ldots, m}$. These current lines are closed through an electric circuit embarked inside the sensor. This circuit measures the vector of the currents $\mathbf{I}=\left(I_{1}, I_{2}, \ldots, I_{m}\right)$ flowing across the $\mathcal{E}_{\alpha=1,2, \ldots, m}$. When an object is placed around the sensor, it generates perturbations of the sensor electric lines portrait that are measured by this current measurement circuit. Finally, the whole device is equipped with an electronic board able to change (in real time) the electric connectivity of the electrodes in order to give to any of the electrodes, the role of $\mathcal{E}_{0}$ in all what has been just said. This ability which we name "electric reconfigurability" allows one to enrich the measurement by changing the previous scalar control input $U$ into a $m \times 1$ vector $\mathbf{U}$. The entire device can be embarked on a mobile body as typically an underwater motion controlled vehicle.

\section{B. Sensor morphology}

Based on this principle, we built a first generation of sensors named "slender probes" due to their high aspect ratio (length/thickness) morphology. On these probes, the macroelectrodes are rings or hemispheres as it is illustrated on the example of Fig. 3 (which is named the 4-electrode probe).

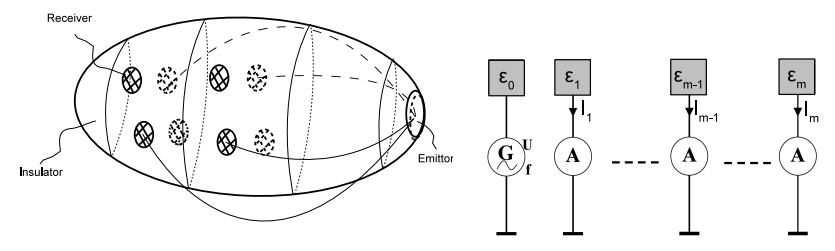

Fig. 2. General principle of the $U-I$ sensor from the external (left) and internal (right) point of view.
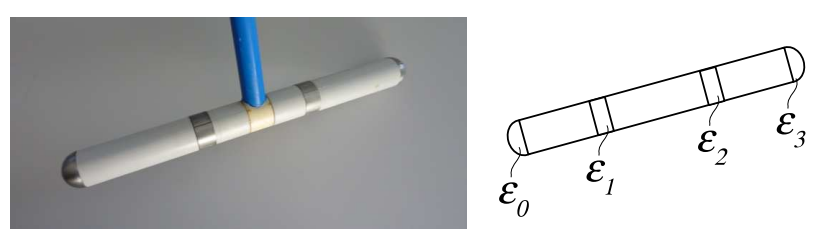

Fig. 3. Picture (left) and schematic view (right) of a 4-electrode sensor.
Based on these design, the sensor has a range of about its body length. This range is that observed on fish of the mormyride family for instance. This limit defines the surroundings of the sensor beyond which the sensor cannot perceive any change in its environment.

\section{AnAlytical Model of MEASURE}

The sensor is approximated by an array of $m+1$ spherical conducting electrodes aligned on a rigid line materializing its solid axis. In the following, the characteristics of the tested sensor are those of Figure 3 with $R=1 \mathrm{~cm}$ for its radius, and $L=22 \mathrm{~cm}$ for its total length. The conductivity of the water is $\gamma=0.04 \mathrm{~S} / \mathrm{m}$ (which corresponds to the ordinary tap water of our lab). By imposing a difference of potentials between each electrode and all the others, an electric field is produced around the sensor. This field produces a field of current lines flowing across the measurements electrodes. The corresponding measured currents are influenced by the presence of objects in the sensor surroundings (Fig. 4-5). In all cases, the effects of the electrically un-transparent objects onto the sensor measurements can be modeled by an expression of the general form (can be numerically done by boundary elements method in [11]):

$$
\mathbf{U}=\mathbf{R}(\mathbf{x}) \mathbf{I},
$$

where $\mathbf{U}$ is the $m \times 1$ vector of the imposed potential differences, $\mathbf{I}$ is the $m \times 1$ vector of the measured currents, $\mathbf{R}$ is a $(m \times m)$ resistance matrix of the scene, while $\mathbf{x}$ is the state vector gathering the (electric and geometric) parameters of the scene. For example, based on Fig. 4, the sphere location with respect to the sensor is parameterized by a distance $d$ and an angle $\theta$, while the parameters encoding the intrinsic properties of the sphere are its electric conductivity $\gamma_{s}$ and its radius $a$. As a result, for this scene the state vector of geometric parameters is: $\mathbf{x}=(d, \theta, a)^{T}$. Now reconsidering (1), the measured currents $\mathbf{I}$ are given by

$$
\mathbf{I}=\mathbf{R}^{-1}\left(\mathbf{x}, \gamma, \gamma_{s}\right) \mathbf{U}
$$

which is highly non linear with respect to the state. Going further, in the following we shall consider the sensor moving in a cubic tank bounded by insulating walls in which are placed a small sphere. In these conditions, the resistance $\mathbf{R}$ offered to the sensor takes the most general form :

$$
\mathbf{R}=\mathbf{A}+\mathbf{S}+\mathbf{W}_{\mathbf{x}}+\mathbf{W}_{\mathbf{y}}+\mathbf{C},
$$

where:

- A models the sensor self resistance, which only depends on the geometry of the robot and is detailed in [8], [12].

- $\mathbf{S}$ models the sphere influence which depends of $d, \theta, a, \gamma, \gamma_{s}$ and is based on a model initially proposed in [13]. A further study of this influence is developed in [8].

- $\mathbf{W}_{x}\left(\mathbf{W}_{y}\right)$ models the wall resistance. It depends on $d_{x}$ and $\theta_{c}\left(d_{y}\right.$ and $\left.\theta_{c}\right)$. A study of this influence based on the so-called "method of images", is developed in [12].

- $\mathbf{C}$ models the influence of the corner which depends on $d_{x}, d_{y}$ and $\theta_{c}$ as detailed in [12]. 
Finally, the model of measurements take the general form:

$$
\mathbf{y}=\mathbf{I}=h\left(\mathbf{x}, \gamma, \gamma_{s}\right),
$$

where $\mathbf{x}$ is the geometric state vector of the sensor surroundings. For the sake of simplicity, in all the following, we assume that: $1^{\circ}$ ) the objects are motion less in the tank frame, $2^{\circ}$ ) the sensor moves in its equatorial plane which is also a symmetry plane (top-down) for the objects; $3^{\circ}$ ) $\gamma$ and $\gamma_{s}$ are a priori known. Thus, the reconstruction only concerns the geometric part of the state $\mathbf{x}$, or "geometric state".

\section{Kalman Filter}

The sensor is put into a tank of our test bench (section $\mathrm{V}-\mathrm{A}$ ) and its (planar) motion is imposed in two kinds of scenarios. In the first set of scenarios, the scene around the sensor is composed of a small object (sphere or cube) in an unbounded conductive fluid (Fig. 4) in which the sensor (or "robot") goes forward in straight line and close to the sphere. In the second type of scenarios, the scene is composed of four walls disposed in a square and of a small object (Fig. 5). In this environment, the robot follows the wall. Finally, in these two situations, the objective of the observer consists in estimating the geometric parameters of the environment gathered into the (geometric) state vector $\mathbf{x}$.

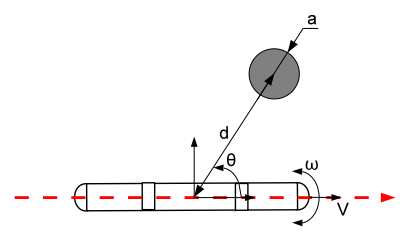

Fig. 4. Simple scene: The sensor moves in an unbounded environment with a sphere, along the red dotted path.

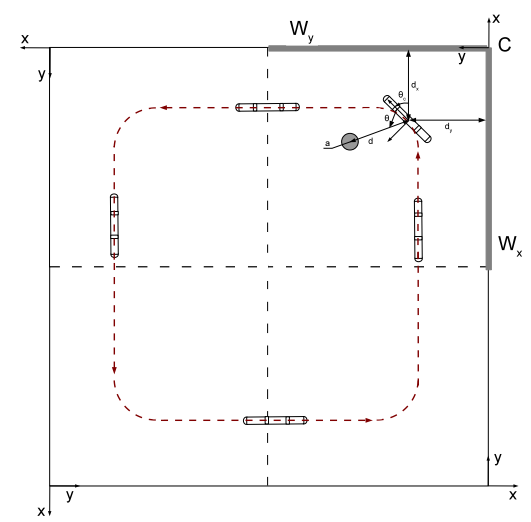

Fig. 5. Complex scenes: The sensor follows the walls of a tank in which a sphere is placed, along the red dotted path.

\section{A. Control and Robot Evolution model}

In our study, the motion of the sensor is imposed. In order to mimic the fish locomotion with a simple model (the fish cannot swim along the lateral dimensions of its body), the sensor motion is modeled as that of a non-holonomic unicycle whose the motions parallel to the $y$ axis are forced to zero. Then the velocities $V_{\|}$(axial velocity) and $\omega$ (yawing velocity) define the vector of control inputs $\mathbf{u}=\left(V_{\|}, \omega\right)^{T}$. In the scenario of Fig. 5, the sphere and the corner (defined by the walls $W_{x}, W_{y}$ and their intersection $C$ ) being fixed in space, the model of evolution of the scene with respect to the sensor can be written under the form:

$$
\left\{\begin{aligned}
d_{x}^{k+1} & =d_{x}^{k}-V_{\|}^{k} \cos \left(\theta_{c}^{k}\right) \Delta t \\
d_{y}^{k+1} & =d_{y}^{k}-V_{\|}^{k} \sin \left(\theta_{c}^{k}\right) \Delta t \\
\theta_{c}^{k+1} & =\theta_{c}^{k}+\omega^{k} \Delta t \\
d^{k+1} & =d^{k}-V_{\|}^{k} \cos \left(\theta^{k}\right) \Delta t \\
\theta^{k+1} & =\theta^{k}+\left(\omega^{k}+\frac{V_{\|}^{k} \sin \left(\theta^{k}\right)}{d^{k}}\right) \Delta t \\
a^{k+1} & =a^{k}
\end{aligned}\right.
$$

where $\Delta t$ is the sampling period, $k$ denotes the time step at which the the state vector,the state vector of geometric variables is then $\mathbf{x}^{k}=\left(d_{x}^{k}, d_{y}^{k}, \theta_{c}^{k}, d^{k}, \theta^{k}, a^{k}\right)^{T}$. In the first type of scenario (Fig. 4), the robot goes forward with an axial velocity $V_{\|}=V_{d}>0$, while its yawing velocity is forced to zero $(\omega=0)$. In any case, the evolution model can be written in the general form :

$$
\mathbf{x}^{k+1}=f\left(\mathbf{x}^{k}, \mathbf{u}^{k}\right),
$$

where $\mathbf{x}^{k}$ is the geometric state vector of the scene and the vector of control inputs is $\mathbf{u}^{k}=\left(V_{\|}^{k}, \omega^{k}\right)^{T}$.

\section{B. Kalman Filter}

The objective of the observer is to estimate the state vector $\mathbf{x}$ of each scene. The chosen observer is an unscented Kalman filter [14]. This choice enjoys the advantages of the classical Kalman filter [15], [16] while avoiding the complex analytical linearization of the evolution equation (6) and mesured model 4. As any Kalman filter, our filter requires to define some matrices which tune its properties. The first one is the covariance matrix $\mathbf{P}_{k}$ which is attached to the state variable $\mathbf{x}^{k}$ and is a trust indicator associated to the estimated state, a small (large) covariance meaning that the state is precisely (imprecisely) known. The evolution of $\mathbf{P}_{k}$ is defined by the unscented Kalman filter algorithm. Two other matrices are defined: $\mathbf{Q}_{m}$, which defines whether the model (6) is well known or not, and $\mathbf{Q}_{s}$ which defines the level of noise on the measurements (4). The principle of the unscented Kalman filter is reminded on Fig. 6. Based on its covariance $\mathbf{P}_{k}$, the filter computes some sampling points (called "sigma points" $\chi^{k+1}$ ) around the estimated state $\mathbf{x}^{k}$ using a deterministic sampling technique known as unscented transfrom. Then, it propagates the sigma points $\chi$ according to: $\left.1^{\circ}\right)$ the evolution model $\chi^{k+1}=f\left(\chi^{k}, \mathbf{u}^{k}\right)$, $\left.2^{\circ}\right)$ the model of measurements $\tilde{\mathbf{y}}=h\left(\chi^{k}, \gamma, \gamma_{s}\right)$, which improves the convergence of the estimated state $\hat{\mathbf{x}}^{k+1}$ and the covariance $\mathbf{P}_{k+1}$.

\section{EXPERIMENTATIONS}

In order to illustrate the performances of the algorithms of object reconstruction and localization, we now report three experiments which have been carried out in a test bench 


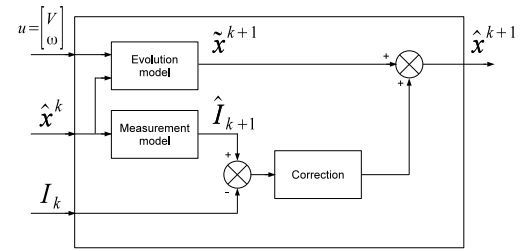

Fig. 6. Principle of the observer.

presented in section V-A. Two of these experiments are carried out in the conditions of Fig. 4, first with an insulating sphere, and second, with an insulating cube. In these two experiments, the small object is modeled by an equivalent sphere whose size and location is estimated. The third (and last) experiment is carried out in the scenario of Fig. 5, where an insulating sphere is placed in a corner while the robot follows the walls of the tank. All, these experiments are carried out with the four-electrode sensor of Fig. 3. The third experiment focuses on the ability of the algorithm to extract the parameters of a small object, once immersed in a complex scene. For each of the experiments, we record the velocity $\left(V_{\|}\right.$and $\left.\omega\right)$ and the measured currents $\mathbf{I}=\left(I_{1}, I_{2}, I_{3}\right)^{T}$.

\section{A. Tank and cartesian robot}

In order to test our electrolocation sensors and algorithms in controlled and repeatable conditions, an automated test bench consisting in a tank of one cubic meter side and a three-axis cartesian robot has been built (see Fig. 7). The robot fixed on top of the aquarium allows probes positioning in translation along $X$ and $Y$ with a precision of $1 / 10 \mathrm{~mm}$ and the maximum speed available is $300 \mathrm{~mm} / \mathrm{s}(\simeq 1 \mathrm{~km} / \mathrm{h})$ for both translations. The orientation in the $(X, Y)$ plane is adjusted in $0.023^{\circ}$ using an absolute yaw-rotation stage and the velocity is $80^{\circ} / \mathrm{s}(13.5 \mathrm{tr} / \mathrm{min})$. The three axes robot is motion-controlled using simulink software with the dSpace system. All probes tested are positioned in the aquarium at adjustable height $(Z)$ using a rigid glass epoxy fibre tube. This vertical insulating tube allows the passage of electrical cables dedicated to the signals coming from the electrodes of the probe. The electrolocation signals are, first processed by analogue chain (amplification and filtering) then converted by a 16 bits ADC (DS2004 card) with a resolution of $0.3 \mathrm{mV} /$ bit (range $+/-10 \mathrm{~V}$ ).

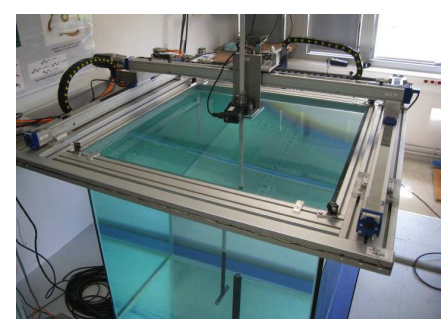

Fig. 7. Electrolocation test bench.

\section{B. Reconstruction of a small object}

In the following, any small object in the surroundings of the sensor is modeled as a sphere whose the size and

location (related to the sensor) are reconstructed by the filter. Before testing the sphere localization, two preliminary tests have been carried out in order to assess whether the modeling assumptions (as replacing any object by a sphere for instance) are justified. All these tests are carried out by

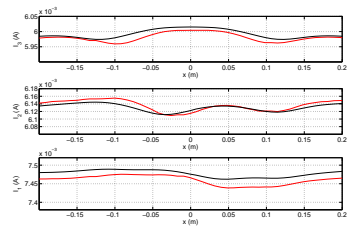

(a) The probe approaches an insulating sphere.

Fig. 8. Measured (red) and modeled currents (black) with the real state vector $\mathbf{x}$ when the probe approaches a small object (sphere model).

comparing the actual measurements with those given by the model (4), once it is informed by the actual geometric (and electric) parameters of the scene. In the first test, a sphere is placed in the middle of the tank and the measurements are recorded in the conditions of Fig. 4. Then, the measurements are computed from the model (4) with the real extended state $\left(\mathbf{x}, \gamma, \gamma_{s}\right)$, and for a scene approximated by the sphere immersed in an unbounded fluid domain. In the second test, the measurements are carried out with a cube located in the middle of the tank, and compared with the same model as in the previous test (a sphere alone in an unbounded world). Here, the sensor goes in straight line with $V_{\|}=0.04 \mathrm{~m} / \mathrm{s}$ and $\omega=0$. The figures 8 (a)-8(b) show the 3 measured currents of the sensor (red) as well as the calculated currents (black) (deduced from the model), when the probe approaches the object. In the conditions of the results of Fig. 8(a), the sphere was insulating and with a radius $a=0.0305 \mathrm{~m}$. The results show that the measured and calculated currents are enough close to each other to consider the model of the sphere enough good for its reconstruction. The plots or figure 8(b) have been obtained with a real insulating cube (of side-length $a=0.040 \mathrm{~m}$ ) located in the middle of the tank, and with the model of a sphere of radius $a=0.037$. The measured and calculated currents are close to each other, so allowing us to conclude that the approximation of a cube by an equivalent sphere is justified.

1) Reconstruction of a sphere in a simple scene (Fig. 4): By "simple scene", we mean that the object (here a sphere) is alone in the sensor surroundings. In this first test devoted to the state reconstruction, the robot moves forward in the tank where an insulating sphere with a radius $a=0.0305 \mathrm{~m}$ is immersed. The initial real and estimated states of the scene are fixed to : $(d=0.257 \mathrm{~m}, \theta=0.294 \mathrm{rad}, a=0.0305 \mathrm{~m})$ and: $\hat{d}=0.146 \mathrm{~m}, \hat{\theta}=0.494 \mathrm{rad}, \hat{a}=0.0105 \mathrm{~m})$. Figure 9(a) shows time evolution of the real (red) and estimated (blue) states of the sphere. Figure 9(b) represents the real (cyan) and the estimated (blue) scenes at different times of the motion. At time $t=2 s$ the sphere is quite good localized but its estimated size is bigger than the actual one. Between 
$2 s<t<8 s$, the estimated sphere is close to the real one. When the object is close to the emitter $(t=8 s)$, the real sphere is well reconstructed and the estimated error is small.

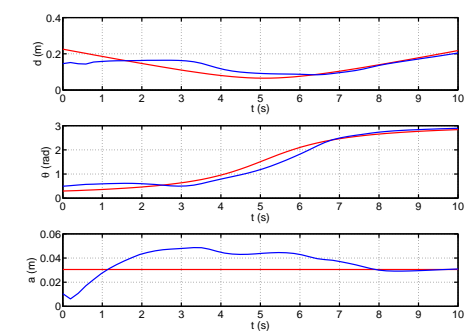

(a) Real (red) and estimated (blue) state of the sphere.
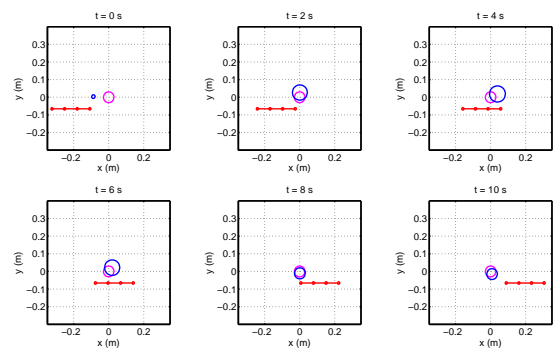

(b) Real (cyan) and the estimated (blue) scene.

Fig. 9. Reconstruction of a sphere in a simple scene $\left(V_{\|}=0.04 \mathrm{~m} / \mathrm{s}\right)$.

2) Reconstruction of a cube in a simple scene (Fig. 4): This second test is carried out in the same conditions as the previous one, except that the object is now an insulating cube. Based on a sphere model, the filter so estimates the parameters of an equivalent sphere. The initial real and estimated states of the scene are fixed to: $(d=0.225 \mathrm{~m}$, $\theta=0.298 \mathrm{rad}, a=0.037 \mathrm{~m}$ (equivalent radius)) and: $(\hat{d}=0.145 \mathrm{~m}, \hat{\theta}=0.498 \mathrm{rad}, \hat{a}=0.001 \mathrm{~m})$. Figure $10(\mathrm{a})$ shows the time evolutions of the real (red) and estimated (blue) state of the cube. Figure 10(b) represents the actual (cyan) and the estimated (blue) scenes at different times of the sensor motion. Like the previous test, at time $t=2 \mathrm{~s}$ the cube is quite good localized but its estimated size is bigger than the side-length of the cube. When the object is close to the emitter $(t=8 s)$, the equivalent sphere is well reconstructed and the estimated error is small. This test illustrates a common feature of all the tests carried out: the estimated equivalent sphere always encapsulates the cube, while its location is well estimated.

\section{Localization and reconstruction of a sphere in a complex scene.}

This test is carried out in the conditions of Fig. 5, with an insulating sphere localized in a corner while the robot follows the wall. This test focuses on the ability of the filter to extract the parameters of a small object in a complex scene. The sphere has a radius $a=0.0305 \mathrm{~m}$. The initial real state of the scene is: $\left(d_{x}=0.3 \mathrm{~m}, d_{y}=0.3 \mathrm{~m}, \theta_{w}=0\right.$ $\mathrm{rad}, d=0.4 \mathrm{~m}, \theta=1.57 \mathrm{rad}, a=0.034 \mathrm{~m})$ and the initial
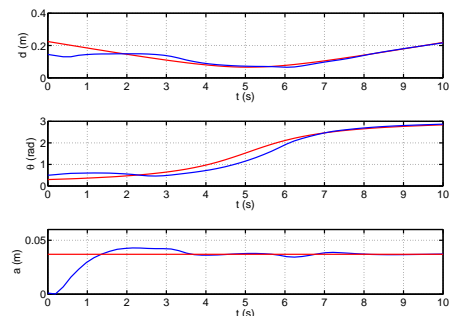

(a) Real (red) and estimated (blue) state of the cube.
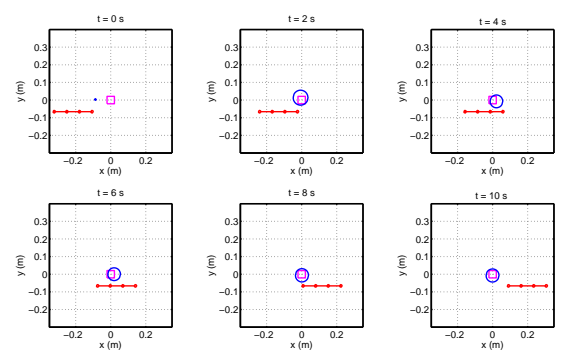

(b) Real (cyan) and the estimated (blue) scene.

Fig. 10. Reconstruction of a cube in a simple scene $\left(V_{\|}=0.04 \mathrm{~m} / \mathrm{s}\right)$.

estimated state of the scene is : $\left(\hat{d}_{x}=0.3 \mathrm{~m}, \hat{d}_{y}=0.3\right.$ $\mathrm{m}, \hat{\theta}_{w}=0 \mathrm{rad}, \hat{d}=0.32 \mathrm{~m}, \hat{\theta}=1.6 \mathrm{rad}, \hat{a}=0.0105$ $\mathrm{m})$. Fig. 11(b) shows the state of the real and the estimated corner with respect to time. Figure 11(a) shows the state of the real and the estimated sphere with respect to the time. Fig. 11(c) represents the actual and the estimated scene at different times of the motion. At $t=0$ the position of the real walls is known (then the initial estimate error for the wall state is null). For the time between $0<t<8 s$, the sensor doesn't detect the sphere because it is too far. At $t>8 \mathrm{~s}$ the sensor perceives the perturbation due to the sphere, then the estimated parameters of the sphere converge to the real ones. For the time $8<t<23 s$, the sensor is close to the sphere and for $t>20 \mathrm{~s}$ the sphere is quite good localized. This test highlights that it is possible to extract (localize and reconstruct) a small object plunged in a complex scene, at least when the sensor is close to this object.

\section{CONCLUdING REMARKS AND PERSPECTIVES}

Using a sensor bio-inspired from electric fish, we shown through experiments: first, that small objects can be reconstructed and localized as equivalent spheres; second, that small objects can be extracted from a complex scene. In spite of these encouraging results, many further works should have to be pursued in future. For instance, let us remark that with the Kalman filter, the current differences between a sphere and a cube are too small to allow the filter to distinguish between them. As a consequence, other strategies more oriented towards the haptic modality of the electric sense could be implemented in order to disambiguate the perception in this critical case. Another way of improvement of the presented result concerns the richness of the sensor 


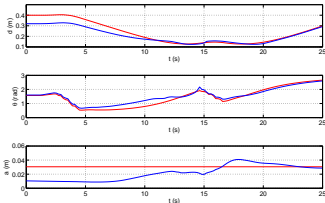

(a) Real (red) and estimated (blue) state of the sphere.

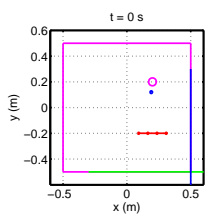

$t=15 \mathrm{~s}$
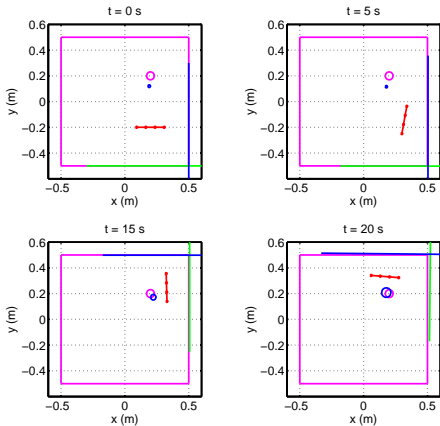

$t=20$
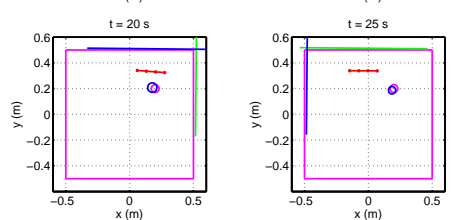

(c) Real (cyan) and the estimated (blue) scene.

Fig. 11. Reconstruction of a sphere in a complex scene $\left(V_{\|}=0.04 \mathrm{~m} / \mathrm{s}\right)$.

measurements. Indeed, with the current sensor design the reconstruction of a sphere (or cube) suffers from intrinsic ambiguity due to the symmetry of the sensor. For instance, two identical objects symmetrically placed on both sides of the sensor generate the same measurements. This scenario is illustrated on Fig. 12, where the estimated state of the scene is initialized to a symmetric position so leading to a reconstructed sphere, symmetric of the real sphere with respect to the sensor axis. In order to disambiguate this kind of situation, we developed a sensor in which electrodes are separated into two lateral (left and right) measurement sub-electrodes. For such a more sophisticated sensor design, the model of the measurements of small objects has been recently proposed in [17]. Among its advantages, this design also allows to identify the electric nature - insulating or conducting - of the objects material, so allowing to release the a priori knowledge of $\gamma$ and $\gamma_{s}$. The implementation of these models in the unscented Kalman filters is today a work in progress. Finally, in the article here presented only the reconstruction problem is addressed. Also, in a second step we should have to include this filter in a global control feedback loop allowing a real underwater robot to navigate in a complex scene. However, we recently proposed another solution to the navigation problem. Based on the embodied philosophy, this alternative solution requires no model of the scene and considers the navigation problem as a low level reflex control problem. Thus, taking benefit of this recent result would allow one to directly couple the reconstructor here presented with this low-level reflex navigator.

\section{ACKNOWLEDGMENT}

The ANGELS project [18] is funded by the European Commission, Information Society and Media, Future and
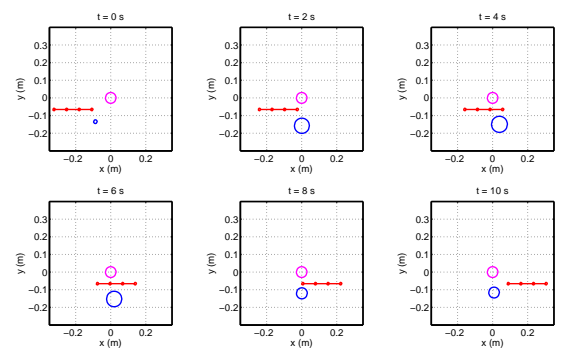

Fig. 12. Real (cyan) and the estimated (blue) scene..

Emerging Technologies (FET) contract number: 231845

\section{REFERENCES}

[1] H. Lissmann and K. Machin, "The mechanism of object location in gymnarchus niloticus and similar fish," The Journal of Experimental Biology, no. 35, pp. 451-486, 1958.

[2] A. Caputi, R. Budelli, K. Grant, and C. Bell, "The electric image in weakly electric fish. ii physical images of resistive objects in gnathonemus petersii," Journal of Experimentation in Biologie, vol. 201, pp. pp. 2115-2128, 1998.

[3] G. von der Emde, S. Schwarz, L. Gomez, R. Budelli, and K. Grant, "Electric fish measure distance in the dark," Letters to Nature, Nature, vol. 395, pp. 890-894, 1998.

[4] $\frac{}{1998}$, "Electric fish measure distance in the dark," Nature, no. 395, 1998

[5] J. Solberg, K. Lynch, and M. MacIver, "Robotic electrolocation: Active underwater target localization," International Conference on Robotics and Automation, 2007.

[6] _ - "Active electrolocation for underwater target localization," The International Journal of Robotics Research, vol. 27, pp. 529-548, 2008.

[7] G. Baffet, F. Boyer, and P. Gossiaux, "Biomimetic localization using the electrolocation sense of the electric fish," in Robotics and Biomimetics ROBIO IEEE, 2008.

[8] V. Lebastard, C. Chevallereau, A. Amrouche, B. Jawad, A. Girin, F. Boyer, and P. Gossiaux, "Underwater robot navigation around a sphere using electrolocation sense and kalman filter," in IROS 2010 IEEE, 2010.

[9] A. Sihvola, P. Ylä-Oijala, S. J. Pää, and J. Avelin, "Polarizabilities of platonic solids," IEEE transactions on antennas and propagation, vol. 52, no. 9, 2004.

[10] N. Servagent, B. Jawad, S. Bouvier, F. Boyer, A. Girin, F. Gomez, V. Lebastard, and P.-B. Gossiaux, "Bioinspired sensor for electrolocation and navigation in conducting media," IEEE SENSORS JOURNAL, vol. submitted, 2011.

[11] M. Porez, V. Lebastard, A. J. Ijsperet, and F. Boyer, "Multi-physic model of an electro fish-like robot : numerical aspect and application to the obstacle avoidance," in IROS 2011, 2011.

[12] B. Jawad, P. Gossiaux, F. Boyer, V. Lebastard, F.Gomez, N.Servagent, S.Bouvier, A.Girin, and M.Porez, "Sensor model for the navigation of underwater vehicles by the electric sense," in Robotics and Biomimetics ROBIO IEEE, 2010.

[13] B. Rasnow, "The effects of simple objects on the electric field of apteronotus," Journal of Comparative Physiology A, vol. 3, no. 178, pp. 397-411, 1996.

[14] J. J. Simon and K. U. Jeffrey, "A new extension of the kalman filter to nonlinear systems," in Int. Symp. Aerospace/Defense Sensing, Simul. and Controls, 1997, pp. 182-193.

[15] R. Kalman, "A new approach to linear filtering and prediction problems," Transactions of the ASME - Journal of Basic Engineering, vol. 82, pp. pp. 35-45, 1960.

[16] H. Nijmeijer and A. V. der Schaft, Nonlinear Dynamical Control Systems. Springer-Verlag, 1990.

[17] F. Boyer, P. Gossiaux, B. Jawad, V. Lebastard, and M.Porez., "Model for a sensor bio-inspired from electric fish," IEEE transactions on robotics (submitted), 2011.

[18] (2009) The angels' project, http://www.theangelsproject.eu. [Online]. Available: http://www.theangelsproject.eu 